\title{
Los problemas de las limitaciones de responsabilidad en el campo de los transportes de mercancías
}

Víctor Humberto Lazo L.

Modernamente, el transporte de las mercaderías por cualquiera de los medios usuales conocidos: transporte terrestre, marítimo o aéreo, o la utilización del moderno transporte multimodal o multipropósito, acarrea frecuentemente problemas para los usuarios, sean éstos embarcadores o consignatarios, que obligan a la presentación de reclamos extrajudiciales o demandas para obtener el resarcimiento del daño causado.

Usualmente se piensa que los directos usuarios de estas vías deben ser solamente los aseguradores; sin embargo, la necesidad de abrir nuevos mercados para los productos peruanos de exportación, así como la demora que a veces se presenta en la atención por el asegurador de los bienes transportados, obliga a que sean los directos afectados los que tengan que iniciar estas acciones.

La ocurrencia de alguna eventualidad, llámese la pérdida o desaparición de los bienes en su totalidad o la avería parcial de éstos, dará lugar a una reclamación por los daños causados en el bien mismo. Adicionalmente, se presentarán también los casos menos frecuentes de acciones derivadas por la demora en la realización del transporte o, lo que es igual, la entrega tardía o cumplimiento defectuoso de la obligación de transporte.

El principio por el cual un transportista está en el deber de responder por aquello que le ocurra a los bienes que recibe, deriva del hecho de que la obligación que éste debe cumplir, consistente en la recepción de una mercadería determinada y su posterior entrega en el lugar acordado, incluye una obligación básica que es la de custodia, convirtiéndose de esta manera el transportista en responsable de lo que le acontezca a esos bienes mientras éstos están en su poder.

Al respecto, comentando la definición de lo que es el contrato de transporte en sí, el jurisconsulto Joaquín Garrigues señala que: «la nota característica del transporte consiste en que el porteador asume directa- 
mente la custodia de la cosa, comprometiéndose a guardarla desde que la recibe del cargador hasta que la entrega al destinatario" ${ }^{1}$.

Esta característica es aplicable tanto al caso de un contrato de transporte puro como a los especiales que están identificados con nomenclatura especial, como es el caso de los contratos de fletamento.

En cualquiera de estas situaciones, pues, se suceden las posibilidades de reclamos, y frente a esta eventualidad el reclamante tiene siempre la esperanza de que sus expectativas serán afrontadas por quien le causó el daño; es decir, de que recibirá un resarcimiento apropiado al daño causado.

Conforme veremos en los párrafos siguientes, esta esperanza en muy pocas oportunidades es amparada, y por el contrario, los transportistas, lamentablemente por la existencia de una legislación que siempre ha estado fuertemente teñida de una influencia decisiva de los organismos que representan a las empresas de transportes, se han visto beneficiados por situaciones excepcionales que no se condicen con la situación obligacional en que se halla el transportista.

En otras palabras, esto significa que, al tratarse de un deudor obligacional que requiere de una experiencia y ciencia especial para desarrollar la labor que se le encomienda, él está generando con su actividad una situación de riesgo excepcional, y que en otras latitudes del Derecho faltas similares son sancionadas con mayor rigor $^{2}$, mientras que por ser el transporte una actividad que despierta un cierto respeto por su antigüedad y la aleatoriedad del servicio prestado, el transportista es tratado por alguna legislación de una manera benevolente. Esta afirmación, de que la actividad del transportista genera una situación de riesgo por la cual debe responder, y que además sirve de fundamento para la determinación de la responsabilidad del porteador, se encuentra magníficamente fundamentada en la cita de Amadeo Soler Aleu, quien tratando el tema señala: "Cuando una empresa realiza y ejecuta, para obtener un beneficio económico, actos encaminados a un objeto cualquiera, asume profesionalmente los riesgos inherentes a esos actos.n ${ }^{3}$.

Conforme veremos en el examen que hemos de realizar, de la amplitud de la responsabilidad que estableció el legislador del Código de Comercio de 1902, tanto para el transporte terrestre como para el marítimo, se ha pasado gradualmente a una limitación económica de su res-

\footnotetext{
Garrugues, Joaquín, Curso de Derecho mercantil, tomo IV, Edit. Temis, Bogotá, Colombia, 1987, p. 207.

Véase al respecto el texto del art. 1970 del Código Civil.

SOler Aleu, Amadeo, Transporte terrestre, Editorial Astrea, Buenos Aires, 1980, p. 73.
} 
ponsabilidad que perjudica gravemente al usuario o acreedor de la prestación, situación que se convierte en un elemento preocupante para nuestro país en particular, en el cual, para el comercio internacional fundamentalmente, los porteadores no se han desarrollado en la proporción esperada y seguimos siendo, quizá por mucho tiempo más, un país fundamentalmente de cargadores de materias primas o semielaboradas, y de recibidores de mercaderías terminadas diversas.

Todo esto, al final, se traduce económicamente en un mayor costo para el país, por cuanto la diferencia que existe entre lo que se puede recuperar de los transportistas y aquello que definitivamente se pierde por culpa de una legislación de este tipo, es trasladada a los usuarios finales o a los aseguradores de carga, quienes, en una política normal traslativa, incrementarán sus primas, incremento que se reflejará en el precio final de los productos que se colocan a disposición de todos nosotros.

\section{Transporte terrestre}

El primer tema que aborda el Código de Comercio de 1902 se refiere al ámbito de la responsabilidad del transportista terrestre. Al respecto debe manifestarse que la manera de tratar este tópico en el Código difiere substancialmente de aquella utilizada cuando se aborda el tema del transporte marítimo, y por cierto, también es muy distante de la que se establece para las otras formas de conducción que hemos señalado.

El principio rector del Código de Comercio, para este caso, es el de la responsabilidad del porteador, excepto en los supuestos de "daños y menoscabos que experimenten los géneros durante el transporte, por caso fortuito, fuerza mayor o naturaleza y vicio propio de las cosas" cuyo caso quien soportará esas pérdidas será el cargador o el propietario de los bienes.

El principio de la exclusión debe ser acreditado por quien invoca la excepción, estableciéndose de esta manera una presunción iuris tantum según la cual, ante la ocurrencia de un evento dañoso, el porteador debe responder, y quien pretende exceptuarse debe demostrar a cabalidad que se ha producido la traslación del riesgo a los hombros del cargador.

Extremando, además, los principios para demostrar la responsabilidad que asume el porteador, el Código señala que, aun en esos casos de excepción en los cuales se le dispensa de la responsabilidad al porteador,

Véase al respecto el art. 356 del Código de Comercio. 
dicha dispensa puede quedar sin efecto «si se probare en su contra que [el caso fortuito, fuerza mayor o naturaleza y vicio propio de la cosa] ocurrieron por su negligencia o por haber dejado de tomar las precauciones que el uso tiene adoptadas entre personas diligentes"s.

Por ahora no entraremos en mayores comentarios sobre estas disposiciones y las glosas que hemos realizado, dejando para otra oportunidad su análisis, que presenta situaciones interesantes.

En los casos en que el porteador pierda los bienes transportados, desapareciendo físicamente éstos y no realizándose la entrega en destino, el Código dispone que aquél debe "pagar el valor que tuvieran los [bienes] no entregados, en el punto donde debieron serlo y en la época en que correspondía hacer su entrega" ${ }^{6}$.

De esta manera se establece el principio de la indemnización por el daño causado, señalándose que el monto retributivo del mismo se fija en función de la especie y no en vista de una cantidad determinada. Sin embargo, es menester precisar que para evitar las sorpresas indebidas el legislador ha determinado a través del artículo 367 del Código de Comercio que esos valores se determinarán con arreglo a lo que se declare en la carta de porte.

Por cierto, y como comentario al margen, resulta muy difícil conciliar el hecho de que el pago indemnizatorio por la pérdida de los bienes se fije en una parte de la ley en función de lo declarado en la carta de porte, que se hace en el puerto de embarque, mientras que de otro lado ese valor debe fijarse, según lo que la misma ley señala, por lo que dichos géneros valían en el puerto de destino y en la época en que correspondía hacer su entrega; es decir, incluyendo ciertos factores que no pueden considerarse objetivamente al inicio, como son: la escasez, las festividades (que incrementan valores), la estacionalidad, etc.

El mismo principio se aplica a los casos de disminuciones de valor por averías, casos en los cuales, según el artículo 359 del Código de Comercio, lo que debe pagar el porteador estará determinado por la diferencia que acrediten peritos entre el valor sano y aquel deteriorado parcialmente; por supuesto, siempre sobre la base de lo que se haya declarado en la carta de porte.

El Código no resuelve el problema frecuente de los casos en que aparecen en las cartas de porte las siglas "NVD" (non value declared o «sin valor declarado"), y si efectivamente se pierden o deterioran éstos, pues no existen términos de comparación que puedan ser utilizados. $\mathrm{O}$ los

Véase en este sentido el art. 357 del Código de Comercio.

Véase sobre este particular el art. 358 del Código de Comercio. 
casos en los cuales por razones de flete o de aduanas se deja de consignar un valor determinado en el efecto porteado.

Es evidente que una cláusula como la indicada por el Código, muy usual en las convenciones internacionales que rigen este tipo de actividades, apareja más complicaciones que soluciones, por lo que debería tenderse a su desaparición, ya que, en todo caso, el valor de un bien o género podrá siempre establecerse en función de su factura de adquisición o mediante la determinación que hagan peritos en caso de imposibilidad de consecución de un documento de esta naturaleza.

No trataremos acá el problema de la responsabilidad por retraso en la entrega, o por mora, que previsoramente el legislador del Código de Comercio introduce en esta parte, y que inexplicablemente no la consideró para el caso de los otros tipos de transportes; pero solamente señalaremos que también se establecen reglas especiales indemnizatorias para esos casos.

El tema de la limitación cuantitativa de responsabilidad para el transportista terrestre aparentemente está dado con amplitud en las citas que hemos realizado, pero, como señala Garrigues, en el pasado todo transportista como depositario de los efectos era responsable de éstos con todo rigor, incluyéndose en este ámbito también los casos de fuerza mayor o caso fortuito; empero, esa rigidez era atenuada limitándose la extensión o quantum de esa responsabilidad.

Producto de la influencia del Derecho civil y de la aparición de las modernas doctrinas de responsabilidad por culpa, la legislación ha impuesto también este criterio para determinar el origen de la responsabilidad, pero equivocadamente ha mantenido la limitación impuesta, lo cual significa una posición de prerrogativa para el porteador, que no se condice con toda la tónica legislativa de nuestro país, e, incluso, del propio Código de Comercio, que no consagra ningún otro caso similar.

La limitación impuesta por la legislación mercantil está dada por el hecho de que solamente se considera, para los efectos indemnizatorios, el valor de la cosa, dejándose de indemnizar la pérdida que ocurra en concreto, la ganancia que se dejó de obtener, limitándose la indemnización al valor de la cosa como tal, señalándose solamente que se trata de un "valor mercantil" o, en otras palabras, según Garrigues, del "precio de venta, como límite normal de la ganancia prevista por el dueño de la mercancía»?

Esta situación descrita es muy similar a la que ahora establece el artículo 1321 del Código Civil, donde se limita en el tercer párrafo el ámbi- 
to de la extensión de la responsabilidad de quien incumple obligaciones, diferenciándolo así de aquel del que las inejecuta con presencia de dolo o culpa inexcusable, en cuyo caso asume una mayor carga de responsabilidad.

\section{Transporte marítimo}

Al tratar este tema nos encontramos con una multiplicidad de alternativas o factores a los cuales podemos recurrir. En primer lugar tenemos el propio Código de Comercio, con su división dual de responsabilidad para el naviero y el capitán. Además tenemos las normas internacionales determinadas por las convenciones internacionales relativas a este tema, de 1924, 1968 y 1978. Por ello, veremos cada una de ellas por separado.

\subsection{Código de Comercio}

La responsabilidad que señala nuestro código para el caso del porteador marítimo, difiere en esencia, en su presentación, de aquellas que señaló originalmente para el transportista terrestre. En efecto, conforme hemos visto en el caso anterior, el Código establece la presunción inmediata de responsabilidad, salvo que se acredite la ocurrencia de alguno de los hechos típicos de exoneración admitidos por el Derecho de obligaciones. Para el caso de del transporte marítimo, también aparentemente por razones sentimentales, se recurre a una nueva fórmula, dividiendo la responsabilidad según se ataque al naviero o al capitán, o según provenga de éstos.

En el caso del naviero, personaje que según el Código puede ser el representante del buque, o el gestor, se establece que éste es responsable, para con los terceros, de "todos los daños que sobrevinieran al [...] cargamento por impericia o descuido de su parte" ${ }^{8}$.

Esto significa que, para el legislador, en el caso del transportista marítimo no existe presunción de responsabilidad por la inejecución de obligaciones, un hecho que ahora está perfectamente determinado por la propia legislación civil para cualquier tipo de contrato', sino que además el afectado deberá acreditar que aparte del daño sufrido éste se de-

Véase sobre este tema el art. 631, inciso 1, del Código de Comercio.

Véase al respecto el art. 1329 del Código Civil. 
bió a un hecho culposo de parte del porteador, convirtiéndose éste, de esa manera, en un sujeto que, por la especialidad de la función que realiza, puede fácilmente quedar exento de responsabilidad ante el desconocimiento que tiene el cargador de las particularidades de su actividad.

Es más, puede llegarse al absurdo que se demuestre un daño, también que no existió fuerza mayor, caso fortuito o vicio propio de la cosa, y que, ante la imposibilidad de establecer la causa propia del daño, se libere de toda responsabilidad a quien tenía la custodia. Así, por ejemplo, el robo parcial de bienes con forzamiento de los sellos podría quedar exento de castigo en el transportista marítimo si no se acredita su impericia o descuido; en tanto que el depositario terrestre está sujeto a una responsabilidad más gravosa ${ }^{10}$, pese a que el usuario tiene, en el último caso, más posibilidades de acceso para efectuar las observaciones pertinentes, que en el primero, en donde se encuentra totalmente alejado y sin posibilidades de algún control inmediato.

La segunda responsabilidad que el Código señala es la del capitán, figura que aparece en nuestra legislación, como lo señala Garrigues, porque se hace una separación entre las obligaciones y responsabilidades propias del incumplimiento del contrato de fletamento (ex-contractu), y aquellas propias de la recepción y custodia de las mercaderías (ex-recepto). Todo esto porque el legislador estimó que "como es el capitán quien recibe la carga, quien expide el conocimiento y quien se compromete a restituirla, es él a quien se impone una rigurosa responsabilidad como depositario, con independencia del contrato de fletamento, aun cuando el capitán no actúe en su nombre, sino en el del naviero que pactó el fletamento" ${ }^{11}$.

Cuando se refiere al capitán, el Código señala sin dudas que la responsabilidad emergente de los actos de éste, que indudablemente se trasladan al naviero por su condición de empleador o real obligado, se basa en el principio de la "falta" o culpa, exonerándolo únicamente de los casos de fuerza mayor ${ }^{12}$. En un caso de falta de técnica legislativa inexplicable, el legislador, para el caso del capitán, sólo incluye el caso de la fuerza mayor como factor excluyente de responsabilidad, mientras que cuando se refiere al porteador terrestre claramente hace los distingos de fuerza mayor y caso fortuito, olvidando, además, también el caso del vicio propio de la cosa como causa de exoneración.

\footnotetext{
Véase sobre este particular el art. 1825 del Código Civil.

Garrigues, Joaquín, véase op. cit., tomo V, p. 321.

Véase sobre este tema el art. 633 del Código de Comercio.
} 
En cuanto se refiere a la extensión que tiene esa responsabilidad, el Código de Comercio repite insistentemente que en ambos casos, una vez acreditada la culpa, deberán responder por "todos los daños" " ${ }^{13}$, sin determinar qué se entiende por tales: si aquellos que se podían prever al tiempo de constituir la obligación -como lo señalaba el Código Civil de 1936 y lo repite ahora el artículo 1321 del Código Civil de 1984, pero limitándolo a los daños emergentes-, o se entiende por "todos los daños" tanto el daño emergente como el lucro cesante, entendiéndose como éstos únicamente aquellos que sean consecuencia directa e inmediata de la obligación, como lo precisa el nuevo Código Civil de 1984.

Esta diferenciación es importantísima, por cuanto cuantitativamente resultaría que para el transportista marítimo se establecería una situación diferenciada con respecto a la de cualquier otro deudor obligacional, sea bajo el Código de Comercio, sea bajo el Código Civil.

Esta exigencia de hacer responsable al porteador marítimo de «todos los daños", no se encuentra solamente en el caso de desaparición o avería parcial de los efectos, sino también en el caso de incumplimiento obligacional en el origen, y así el artículo 627 del Código de Comercio responsabiliza de igual manera al capitán por la inejecución de un viaje sin que medie «accidente fortuito o caso de fuerza mayor que se lo impida" ${ }^{14}$, lo cual apunta indudablemente a considerar que el principio de extensión lata fue el que estuvo presente en el legislador de 1902 con respecto al acarreo marítimo.

Otro tema que surge como consecuencia de esta imprecisión legislativa es el hecho de que al sancionar de manera vaga con «todas las consecuencias" no se está diferenciando los casos de incumplimiento derivado del dolo o culpa inexcusable, de aquellos procedentes de la culpa simple. Éste es un hecho claro que, definitivamente, no puede atribuirse a desconocimiento del legislador sobre las diferencias existentes entre los casos ya anotados, pues el Código Civil de 1852 sí las consideraba, y además, doctrinariamente no se trata de una concepción reciente, sino que data desde el Derecho romano y de los post-glosadores.

Tratando de explicar esta incongruencia, lo único que podría pensarse es que el legislador estimó que en el tipo de obligaciones a cargo del transportista, que involucra la recepción, cuidado, conservación y reentrega de los bienes, la violación de cualquiera de estos deberes esenciales no podía ser considerada como procedente de la culpa leve, sino

\footnotetext{
1.3 Véase al respecto el artículo 631, inciso 1, del Código de Comercio.

14 Se incluyen también como excepciones los casos estipulados en el inc. 2 del art. 702 y los casos del art. 703 del Código de Comercio, aun cuando no se mencionen por concordancia.
} 
que en todo caso debía ser tipificada siempre como culpa grave debido a la imposibilidad de parte del usuario de intervenir o cautelar adecuadamente sus intereses. En todo caso, este tema queda solamente para la reflexión tanto jurídica como jurisprudencial, pues no existen datos o caminos que despejen esta interrogante.

Un punto que no debe ser olvidado, y que mueve también a reflexión, es la carencia de incidencia, en la responsabilidad del porteador marítimo, de la mora en el cumplimiento de la prestación ofrecida, aspecto que sí ha estado presente en el caso del transportista terrestre y que con frecuencia aparece en las relaciones de este tipo, pero que el Código inexplicablemente silenció.

\subsection{Convención internacional de 1924}

Toda esta situación cambia radicalmente cuando se aprueba en Bruselas, Bélgica, la Convención internacional para unificación de ciertos términos relativos a conocimientos de embarques, aprobada el 25 de agosto de 1924, instrumento multinacional que fue aprobado por el Perú mediante la resolución suprema n. ${ }^{\circ} 687$ del 16 de octubre de 1964, quien extendió el instrumento de adhesión respectivo el 17 de octubre del mismo año y lo depositó ante el gobierno de Bélgica el 29 de octubre de 1964, entrando en vigencia en el Perú desde el 29 de abril de $1965^{15}$.

Esta convención, que es popularmente conocida como las «Reglas de La Hayan, establece nuevamente el principio de la culpa como base para la responsabilidad del transportista en los casos en que se utilizan conocimientos de embarque, así como una lista de exoneraciones favorables al transportista, sin siquiera considerar en muchas de ellas la posibilidad de concurrencia de la misma culpa.

Sin entrar a analizar estos temas, que bien pudieran ser objeto de un próximo trabajo, nos limitaremos a señalar las bases de limitación que se incluyen en esta convención, la cual se basa exclusivamente en el aspecto valorativo de la cosa dañada o desaparecida, omitiendo también expresamente toda referencia al tema de la demora en la entrega y al de los daños que pudieran ocurrir.

15 La incorporación legislativa que se ha hecho de esta convención internacional ha sido seriamente objetada en un trabajo del Dr. Alfredo Ostoja L. A., en el cual se demuestra que se afectaron principios constitucionales de la Constitución de 1933 y que por ende ese documento es objetable. No obstante este hecho, trataremos el tema sin tener en cuenta esta objeción y considerando que es una norma válida, particularmente por incorporarse muchas veces de otra manera en los contratos que se celebran, al ser una convención ampliamente difundida. 
La Convención señala en su inciso 5 del artículo 4 , que «ni el transportador, ni la nave, en caso de pérdida o daño causado a las mercaderías o en lo concerniente a ellas, serán responsables por suma que exceda de 100 libras esterlinas, por bulto o unidad [...], salvo que la naturaleza y el valor de dichas mercaderías hubiesen sido declaradas por el cargador antes del embarque [...]».

El primer tema que llama a reflexión, en orden inverso, es que la Convención nuevamente señala, aunque esta vez de una manera más técnica que nuestro vetusto Código de Comercio, la posibilidad de incrementar la responsabilidad. Lamentablemente no se previó en la norma qué pasaba en los casos en que el porteador no facilitara el cumplimiento de este sistema, situación que ocurre en la práctica, pues ningún conocimiento de embarque tiene un espacio para la declaración del valor de la carga.

Ahora bien, en cuanto al tema de las 100 libras esterlinas, siempre ha sido de criterio generalizado que la unidad monetaria a que se refiere esa convención era la de las libras esterlinas de uso actual y conocido, es decir, la moneda de vigencia en el Reino Unido. Sin embargo, lo que la Convención señaló fue una situación muy diferente. Efectivamente, el artículo 9 de la citada convención estableció que «las unidades monetarias de que se trata en la presente Convención se entienden de un valor en oro", y, por ende, debe determinarse de qué valores de equivalencia se está tratando.

La Convención fue aprobada en 1924 y en esa época la moneda de uso corriente en el Reino Unido estaba referida al patrón oro, existiendo por tanto la libra de oro inglesa de igual manera como había existido en el Perú, después de la guerra del Pacífico, la libra peruana de oro, que todavía es utilizada por algunas damas como apéndice decorativo de esclavas y collares. Recién después del famoso "crac" de la bolsa de Wall Street, de 1929, que afectó a toda la economía mundial, es apreciada la necesidad de proteger las reservas de oro de los países; e Inglaterra, en setiembre de 1931, declaró la inconvertibilidad de la libra esterlina de oro y el uso legal y forzoso de la libra de papel, como se conoce hasta nuestros días ${ }^{16}$.

Una libra de oro inglesa era una moneda con un contenido determinado de oro de 916 2/3 milésimas de oro. La ley Coinnage Act de 1971, emitida en Inglaterra, claramente señala que el contenido oro de 100

\footnotetext{
16 Sobre este tema puede verse el libro de Domingo LoPez SAAVEDRA, Responsabilidad del transportista por agua, La Ley, Sociedad Anónima Editora e Impresora, Buenos Aires, 1971, p. 73 y ss.
} 
libras esterlinas o soberano es de 798,805 gramos, de una pureza de 916,66 milésimas, que por no ser íntegramente de oro, como ya hemos señalado, sólo alcanzaba a 916 milésimas, resultando por conversión una moneda con solamente 732,238 gramos de oro puro.

La onza troy que se utiliza como medida para valorizar el oro de pureza equivale a 31,103 48074 gramos, y su valor es fácilmente determinable día a día por ser un metal de cotización mundial. De acuerdo a las publicaciones que aparecen diariamente en diferentes diarios de la capital, sabemos que el valor de la onza troy ha alcanzado durante el pasado mes de mayo de 1994 el valor de US\$ 385 en los mercados internacionales de Londres, New York y Zurich (en el mercado nacional bordea los US\$ 350), lo cual significa que para determinar el monto máximo de la responsabilidad que está señalando la Convención sólo será necesario realizar una simple operación de regla de tres expresada de la siguiente manera:

$$
\begin{aligned}
& 31,103-\text { US } \$ 385 \\
& 732,238-\mathrm{X} \\
& \text { O, lo que es lo mismo: }
\end{aligned}
$$

$$
\frac{732,238 \times 385}{31,103}=\text { US\$9063,80 }
$$

Esto nos lleva a establecer, sin ninguna duda, que el valor equivalente de cada bulto o unidad de medida, para los efectos de la Convención, alcanza a US\$ 9063,80 aproximadamente, lo cual está muy lejos de las 100 libras esterlinas en valor papel, que muchas veces se pagan, así como de las equivalencias que lamentablemente se han hecho inmutables en otras legislaciones y que se han fijado en cumplimiento del derecho que señala la propia Convención: en EE.UU., \$ 500; en España, 5000 ptas; en Alemania DM 1 250; en Holanda 1250 fls., por citar solamente algunos $\operatorname{casos}^{17}$.

En el Perú, el único caso reportado en el cual se trató el tema de esta limitación de manera puntual es el que se decidió en la Corte Suprema

\footnotetext{
1 La aplicación de estos principios no es solamente doctrinaria o teórica, pues similar forma de pensar se ha alcanzado en las cortes de Inglaterra, Italia, Francia, Canadá, India, Singapur y Argentina, en las que se ha fijado una cuantificación más elevada para la equivalencia de esa cifra señalada por la Convención.
} 
de la República el 17 de julio de 1992, en la causa 2133-88 seguida por Heinrich Schdmit contra Empresa Naviera Santa S.A., sobre pago de dólares ${ }^{18}$. En este caso, la Corte Suprema, tratando el tema de la limitación de la responsabilidad, precisó: "que, sin embargo, el artículo noveno dispone que las unidades monetarias de que trata dicha Convención se entienden en valor oro, y en aquellos Estados contratantes en los que la libra esterlina no sea empleada como unidad monetaria, se reservan el derecho de convertir en cifras redondas, según su sistema monetario, las sumas indicadas en libras esterlinas en la mencionada Convención; que, en este sentido, la demandada que ha invocado el límite de responsabilidad en su favor no ha probado cuál es el valor en oro de las cien libras esterlinas a que se refiere la Convención, ya que la Resolución Suprema antes acotada, que aprueba las Reglas de la Haya, no establece su equivalencia en moneda nacional ni los mecanismos para fijarla [...]».

Es interesante anotar solamente, como comentario al margen, que pese a que la Corte Suprema determinó que no era la libra papel sino la conversión oro la que debía urilizarse, y aun cuando reconoció que era una norma de obligatorio cumplimiento, no hizo las indagaciones respectivas que hubiesen sido deseables para de una vez fijar un patrón que debiera seguirse en el futuro. Existen otras consideraciones o conclusiones que pueden derivarse de esta ejecutoria pero que por ahora no abordamos por no pertenecer al tema que estamos tratando.

\subsection{Convención de 1968}

La Convención internacional de 1924 fue objeto de revisiones posteriores, hasta que finalmente se arribó al protocolo modificatorio suscrito en Bruselas el 23 de febrero de 1968, conocido mundialmente como las Reglas de La Haya-Visby, en el cual a través de catorce artículos se varían substancialmente aspectos cuestionables de la anterior legislación, y en particular el tema referido a la extensión cuantitativa de la responsabilidad.

Mediante el artículo 2, inciso a, precisó una nueva forma de delimitar el máximo indemnizatorio, y fijó que ni el transportador ni el buque serán responsables en ningún caso por pérdidas o daños causados a las mercaderías, por una suma superior al equivalente de 10000 francos por bulto o unidad.

18 Reportada en Jurisprudencia Civil, de Normas Legales S.A., 1993, pp. 62 y 63. 
Este franco al que se refería la Convención ${ }^{19}$ se encuentra definido en el inciso $f$ del mismo artículo como una unidad consistente en $« 65,5$ miligramos de oro del título de 900 milésimos de fino». Esto significaba que los 10000 francos equivalían a 655 gramos de oro que no alcanzaban la pureza absoluta, pues, conforme hemos citado, la propia Convención establece que faltaba $1 / 10$ para alcanzar esa calidad; de allí que si queremos tener un oro puro debemos restar esa diferencia, lo cual nos arroja una equivalencia de solamente 589,5 gramos de oro puro, que es notablemente inferior a los 732,238 gramos que contenía un soberano de oro equivalente a 100 libras de oro.

Considerando los mismos valores ya conocidos del caso anterior, esta moneda que establece la Convención significaría, a los mismos valores de onza troy ya identificados, lo siguiente:

$$
\frac{589,5 \times 380}{31,103}=7202,19
$$

En otras palabras, el hecho de haber transcurrido cuarenta y cuatro años de la convención original llevó a los nuevos redactores a disminuir su valor en vez de considerar que por efecto natural del incremento inflacionario los costos habían subido, y así en ese lapso se perdió un $19,5 \%$ de equivalencia, en favor de los transportistas, mientras que la economía mundial en ese mismo lapso había sufrido notables cambios, desapareciendo el patrón oro del ámbito económico de todos los países del orbe, producto de la crisis monetaria americana.

\subsection{Convención de 1978}

El último cambio con relación a la extensión de la responsabilidad del transportador marítimo ocurrió con la recientemente vigente Convención de Hamburgo, suscrita el 31 de marzo de 1978 en la ciudad alemana del mismo nombre. La dación de esta convención fue producto de un intento de UNCTAD y UNCITRAL, ambos, organismos de las Naciones Unidas, por mejorar la situación de los países en desarrollo «a

19 El franco de la Convención es también conocido como «franco Poincaré» y es una moneda ideal que por primera vez aparece en el texto de la Convención de Varsovia de 1929, que rige el transporte aéreo internacional, y su valor equivalente en oro es exactamente el mismo que el fijado por esta convención. 
través de un incremento de la responsabilidad del transportador dentro de una adecuada distribución de riesgos, sin que ello significara un aumento de los costos de los fletes". ${ }^{20}$

El resultado no fue todo lo optimista que se pudiera pensar, pues, conforme podremos apreciar del mismo documento, lo que se obtuvo fue un aparente avance, incluyéndose la responsabilidad por demora, hasta ese momento inexistente, pero con un notable retroceso en cuanto se refiere a la limitación de la responsabilidad.

El Convenio en su artículo 6, primer párrafo, a, limita la responsabilidad del transportador a una suma equivalente a 835 unidades de cuenta por bulto o unidad de carga transportada. El artículo 26 precisa que esa unidad de cuenta no es otra que el derecho especial de giro definido por el Fondo Monetario Internacional. La mecánica de la conversión que usamos nos lleva a la inevitable conclusión de que la limitación que ya conocíamos de la Convención de Bruselas de 1924 queda reducida a su mínima expresión, y que, en resumidas cuentas, un porteador, cincuenta y cuatro años después, en vez de pagar un monto mayor por el daño que puede haber causado por la ejecución defectuosa del contrato de transporte, solamente responderá por aproximadamente la novena parte de ese valor originalmente estipulado ${ }^{21}$.

La cuantificación de lo anteriormente señalado se representa de la siguiente manera:

\section{5 unidades $\mathrm{x}$ US $\$ 1,2=$ US $\$ 1002$}

La limitación de responsabilidad aceptada en 1924 alcanzaba, como ya hemos señalado, US\$ 9063,80 aproximadamente, lo cual significa que ahora por el mismo bulto, setenta años después, un transportista pagará por su desaparición o por averías un máximo de nueve veces menos, y eso, sin considerar que los dólares de 1924 permitían adquirir más bienes que esa misma cifra trasladada a la época actual, todo producto del efecto inflacionario y económico mundial.

\footnotetext{
López SaAvedra, Domingo M., citado por Basaldúa Romero, Luis, en Responsabilidad del transportador de mercaderias por agua, Editora Córdoba, Argentina, 1985 p. 200.

2 La unidad monetaria denominada "DEG" o "derecho especial de giro", resulta de promediar las seis monedas que registren un menor índice de fluctuación y pertenezcan a los países de mayor grado de desarrollo. En la actualidad el valor de cada DEG alcanza aproximadamente a un equivalente de US $\$ 1,2$, que es similar al que tenía en el momento de su creación. La paridad DEG oro existió en un primer momento, pero fue posteriormente eliminada por una enmienda del FMI.
} 


\section{Transporte aéreo}

El siguiente tema que requiere ser analizado desde el punto de la extensión de la responsabilidad del porteador, es el relativo al transporte aéreo.

En el caso de nuestro país, recientemente nos hemos incorporado debidamente a la legislación internacional al haber ratificado todo el grupo de normas que rigen internacionalmente el transporte aéreo, y en particular el Convenio de Varsovia de 1929, que rige de manera preferente las relaciones entre todos los países usuarios del transporte de este tipo.

En efecto, mediante la resolución legislativa n. ${ }^{\circ} 24819$ del 26 de mayo de 1988, el Perú accedió a todos los tratados internacionales que integran el régimen de Varsovia, adoptándose, por ende, por el Perú el Convenio de Varsovia del 12 de octubre de 1929, el Protocolo modificatorio de La Haya del 28 de setiembre de 1955, las modificaciones I y II de Montreal, o Acuerdos de Montreal, y el Protocolo de Guatemala del 8 de marzo de 1971.

Los efectos de esta legislación internacional, parte de cuyas normas no están aún en vigencia a nivel mundial por faltar todavía el número requerido de países signatarios, han hecho cambiar dramáticamente el campo de la responsabilidad aeronáutica en el Perú en cuanto se refiere al transporte internacional, que es el que hemos estado tratando hasta ahora.

Hasta antes de esta integración del Perú al ámbito legislativo universal, lo cual ha ocurrido con cincuenta y nueve años de retraso, el Perú estaba regido por la ley de Aeronáutica Civil n. ${ }^{\circ} 15720$ del 11 de noviembre de 1965 y su reglamento, el decreto supremo n. ${ }^{\circ} 16$ del 28 de diciembre de 1965. Estas disposiciones establecieron de manera inmutable una limitación de ascendente a un máximo de S/. 200,00 (doscientos soles antiguos) por cada kilogramo, y de $S / .5$ 000,00 (cinco mil soles antiguos) por cada unidad de equipaje.

Considerando los efectos inflacionarios que sufrió el país, se podrá apreciar que estas cifras eran poco menos que ridículas. Basta señalar que a los dos años de su dación se produjo una devaluación traumática del dólar, que pasó de S/.27,00 a S/.43,38, es decir, casi el doble, con sus consiguientes efectos multiplicadores en la economía y devastadores en lo que recibirían los afectados por los actos de los transportistas. Es historia conocida después, lo ocurrido durante la década 1980-1990 con el aniquilamiento de la moneda nacional, su cambio por otras unidades monetarias y naturalmente la manera como esto afectó los derechos de aquellos usuarios del transporte aéreo que se vieron afectados por pérdidas o daños en sus efectos. 
El cambio por el sistema de Varsovia significó evidentemente un alivio y un progreso en la limitación de responsabilidad impuesta en favor del porteador aeronáutico. Esta norma -nos referimos al Tratado internacional para la unificación de ciertas reglas relativas al transporte aéreo internacional, suscrito en Varsovia el 12 de octubre de 1929, a través de su artículo 22, que ha sufrido diversas enmiendas y modificaciones- fijó que la limitación de responsabilidad, en los casos de transporte de mercaderías, quedaba circunscrita a "[...] la cantidad de 250 francos por kilogramo". Los francos a los que se refería la Convención -y ahora ley nacional por ser un protocolo vigente a nivel internacional-, era, según el inciso 4 del mismo artículo, un "[...] franco francés constituido por 65,5 miligramos de oro con ley de 900 milésimos de fino [...]”.

Esto significaba que por cada kilogramo de cargo el porteador sólo estaba obligado a pagar un total de 16,25 gramos de oro de 900 milésimos de fino, por lo que, convertido a oro puro de 1000 milésimos, el total arroja 14,62 gramos $^{22}$.

Si tenemos en cuenta que la onza troy, como ya hemos indicado, contiene 31,103 gramos, resulta que esta limitación por cada kilogramo representa el $47 \%$ del valor asignado a la onza troy. Como sabemos, este metal tenía para el mes de mayo de 1994 en los mercados de Londres, New York y Zurich, donde normalmente se transa, un precio de US\$ 385,00 , lo cual implica que la limitación del porteador aéreo, según esta norma, estaba en US\$180,95 por kilo. Una cantidad bastante lejana de los usuales US $\$ 20,00$ por kilogramo que las empresas aéreas acostumbran a pagar como máximo de limitación de responsabilidad, monto que como veremos más adelante, nace de sus presiones, necesidades y de un nuevo protocolo aún no vigente, que inexplicablemente el Perú también suscribió.

Producto de la situación económica caótica de Estados Unidos de América en la década de 1960, que lo obligó a abandonar el patrón oro, del alto incremento de los intereses en su mercado interno y el "boom" petrolero de 1973, el precio del oro alcanzó valores inimaginables, llegando en las bolsas internacionales a cotizarse hasta en cerca de US\$ 900,00 la onza troy, es decir, casi dos veces y medio su actual valor. Los transportistas internacionales iniciaron una fuerte presión internacional para variar este patrón de responsabilidad, argumentando que afectaba sus seguros, los costos y que era necesario para la estabilidad mundial el buscar una solución segura, integral y uniforme.

\footnotetext{
Todas las operaciones que se han realizado son aproximadas y redondeando las cifras para mayor facilidad e ilustración. El oro de 1000 milésimos puro absoluto no existe, y normalmente se trabajó a un máximo de 916,66 milésimos.
} 
El producto de esta presión fue el recurrir a los famosos Derechos Especiales de Giro (DEG) del Fondo Monetario Internacional, ya explicados, y consiguieron estos transportistas aéreos, a través de sus gobiernos, que se suscribiese el Protocolo de Montreal I del 25 de setiembre de 1975 y el anexo del Protocolo II, que ahora el Perú también ha aprobado y ratificado, por el cual se variaron los límites de responsabilidad señalados en el artículo 22 de la Convención de Varsovia del 12 de octubre de 1929 .

El nuevo límite escogido, también de manera arbitraria, fue de 17 DEG por cada kilogramo de carga averiada o perdida. El valor de un (1) DEG, cuando éste se creó, era de US\$ 1,2, lo cual ocurrió hace aproximadamente veinte años. En la actualidad se mantiene en la misma paridad de equivalencia o ha mejorado a lo más 0,10 .

El resultado de este nuevo cambio dio como efecto el reducir el límite de la responsabilidad, de US\$180,95 por kilo, según el Protocolo de Varsovia de 1929, a US\$20,4 según el Protocolo modificatorio de Montreal de 1975. En sólo cuarenta y seis años la responsabilidad de los porteadores aéreos había descendido $88 \%$, en vez de crecer. Los precios de la economía mundial en ese mismo lapso no han descendido; por el contrario, han crecido.

A los efectos de darle inmediata vigencia a este protocolo, que no se encuentra todavía en efectividad a nivel mundial, las líneas de transporte aéreo reunidas en la IATA (International Airline Transportation Association), acordaron, ilegalmente, pagar en adelante solamente los famosos US $\$ 20,00$ por kilogramo, que son conocidos por todos aquellos que alguna vez han tenido un percance de daños o pérdida de carga.

El Gobierno del Perú, no sabemos si advertida o inadvertidamente, cuando decide integrarse a la comunidad internacional legislativa en materia de aeronáutica, aprobó, en la famosa resolución legislativa ya mencionada, estos Protocolos de Montreal de 1975, que favorecen a los porteadores y desfavorecen a un país tradicionalmente cargador, como es el nuestro.

Curiosamente, en la resolución legislativa mencionada no se incluyó como aprobado el Acuerdo de Montreal de 1966, que, modificando los principios de responsabilidad por culpa en caso de muerte o heridas a pasajeros, obliga a los transportistas a reconocer un límite de US\$ 75000,00 por pasajero, sin que sea posible exceptuarse en forma alguna al transportista, modificando tal acuerdo el Protocolo de Varsovia, el que para esos casos, si bien es cierto fijaba un máximo de 125000 francos oro, que representan aproximadamente US\$ 91212 por pasajero como monto máximo -que luego se duplicaron por la Convención de La Haya de 28 de setiembre de 1955-, permitía reducir tal máximo 
limitativo e, incluso, exonerar todo pago, si el porteador demostraba que "[...] él y sus representantes adoptaron todas las medidas necesarias para evitar el daño o que les fue imposible adoptarlas" ${ }^{23}$.

Si recurrimos al campo de la responsabilidad para el transporte interno, en materia aeronáutica, apreciamos que la situación de limitación de responsabilidad es poco menos que ridicula, producto de utilizar valores convencionales limitativos. La ley de Aeronáutica Civil n. ${ }^{\circ} 24882$ de julio de 1989, fija como monto máximo de responsabilidad por muerte de pasajero peruano en un vuelo nacional 20 Unidades Impositivas Tributarias (UIT), es decir, a valores actuales, S/. 34000 , o, lo que es lo mismo, US\$ 15600 por cada accidentado peruano dentro del territorio nacional. Curiosa discriminación de la ley. Un pasajero nacional que viaje en un avión peruano al extranjero y que fallezca en un accidente puede recibir hasta US\$182 424. Pero si el mismo pasajero nacional, volando en la misma aeronave, fallece en un accidente en un vuelo nacional, recibirá solamente 1/12 (un doceavo) de lo que recibiría en un vuelo internacional por el mismo hecho. Justicia extraña.

En materia de daños a la carga, la situación no es diferente: el porteador nacional solamente está obligado a pagar un máximo de $4 \%$ de la UIT por kilogramo. En cifras simples, eso significa S/. 68,00 por kilo, que convertido en moneda americana arroja US $\$ 31,19$, bastante alejado de los US\$180,95 que fija la misma ley como limitación si el daño ocurre en el caso del transporte internacional.

\section{Transporte multimodal}

El último de los aspectos que nos queda por revisar en este viaje por los diferentes montos máximos o limitativos de responsabilidad que contiene la legislación nacional sobre el transporte, es el relacionado con el transporte multimodal. Éste es un tipo de actividad moderna que tiene gran auge en los países desarrollados y que cada vez integra más a nuestro país al mundo del transporte internacional.

El transporte multimodal está regulado en el Perú por el decreto legislativo n. ${ }^{\circ} 714$ del 7 de noviembre de 1991 . Esta norma, que reemplazó al decreto legislativo n. ${ }^{\circ} 684$ expedido días antes -por existir graves incongruencias en el aspecto de jurisdicción aplicable, al haberse transcrito las disposiciones del proyecto de la UNCTAD del 24 de mayo de 1980

23. Véase el artículo 20, inciso 1, de la Convención de Varsovia de 1929. 
sin haberse concordado con la legislación interna-, define en su artículo 1, párrafo 1, como transporte multimodal: "el porteo de mercancías utilizando por lo menos dos modos diferentes de transporte, desde un lugar situado en un país en que el operador del transporte multimodal toma las mercancías bajo su custodia, hasta otro lugar designado para su entrega situado en un país diferente».

La primera reflexión que origina la lectura de la parte introductoria de esta norma, donde se hallan las definiciones, es que producto de la no adopción por el país del Convenio de las Naciones Unidas sobre transporte internacional multimodal de mercancías, adoptado en la Conferencia del 24 de mayo de 1980 , tratado que no se encuentra vigente a nivel mundial aún, se ha incorporado en el país un conjunto de normas que sirven para regular exclusivamente el transporte multimodal internacional, pero no el que se pueda generar nacionalmente, porque su ámbito de aplicación definido por el artículo ya citado obliga a que la labor de porteo se haga de un país a otro.

De este modo, la posibilidades de transporte multimodal que se hace con la región de la Selva a través usualmente de camiones y transporte fluvial, o incluso con la Sierra, por buques y camiones, quedan totalmente desprotegidas y sin normatividad, creando de esa manera una dicotomía en el ámbito de la responsabilidad, pues, en el caso de un transporte internacional existe limitación única, y en el caso del nacional, una limitación en función del transporte utilizado o sistema "red".

En lo que se refiere a la responsabilidad en sí del operador de transporte multimodal u OTM, como lo denomina la norma ya señalada, se ha establecido por el legislador en el artículo 19 del dispositivo indicado, que aquél tiene derecho a limitar su responsabilidad por «[...] cualquier pérdida o daño [a] una suma que no exceda al equivalente a 666,67 SRD (unidades especiales de giro) por paquete o unidad, o a 2 SRD por kilo de peso bruto de las mercancías perdidas o dañadas, si esta cantidad fuera mayor».

El primer comentario que suscita este artículo es el hecho de que se cita como unidad de cuenta para los efectos de la limitación, según el artículo 1, párrafo 10, a las «unidades especiales de giro»; sin embargo, se les abrevia como SRD, que corresponden a la expresión en inglés "special drawing rights" y no a "derechos especiales de giro" o DEG, como se les conoce en todos los países de habla hispana. Como reflexión curiosa debe mencionarse que la norma indica que éstos son "la unidad de cuenta tal como ha sido definida por el Fondo Monetario Internacional (copiar la definición del FMI)" (sic). Por supuesto, al no haberse copiado en la ley la definición, la famosa unidad de cuenta legislativamente se encuentra coja. 
El segundo comentario que motiva esta norma es el hecho de que limita la responsabilidad a la suma ideal que allí se señala por "[...] cualquier pérdida o daño [...]", lo cual incluiría todo tipo de comportamiento de parte del operador del transporte multimodal. Sin embargo, el artículo 13 señala que no existe tal limitación en los casos en que aquél actúe dolosamente haciendo constar información falsa sobre las mercancías u omita cualquier información que debía indicarse en el documento de transporte multimodal.

Eso significa que solamente en el caso en que, al momento de configurarse el documento que prueba la existencia del contrato de transporte multimodal, se haya comportado el OTM dolosamente, perderá esa limitación de responsabilidad. Pero ¿̨qué ocurre si su comportamiento no es doloso -el dolo importa una noción subjetiva de intencionalidad-, sino solamente de culpa grave? Al no existir diferenciación en la ley, y aplicando el principio del numeral IV del Título Preliminar del Código Civil, dicho OTM, que se comporta de esa manera, seguirá contando con el beneficio de la limitación.

La redacción legislativa es deficiente, pues en vez de acumular en un solo campo la pérdida de los derechos protectivos por actos derivados de dolo o culpa inexcusable en la etapa de formación del contrato y en la de su ejecución, separa éstas en dos artículos ( 13 y 25), contemplando dolo sólo para un caso, y dolo y culpa grave para el otro. Es más, la forma de definición que utiliza el artículo 25 para los casos de daño causado por dolo o culpa inexcusable es reiterativa y llama a confusión, porque estos conceptos están claramente definidos en nuestra legislación civil en los artículos 1318 y 1319 del Código Civil, no obstante lo cual, luego de urilizar estos conceptos, el artículo 25 agrega: «o de una acción u omisión del OTM realizada con intención de causar tal pérdida, daño o retraso o temerariamente y a sabiendas que probablemente los mismos se produjesen".

Cabe preguntarse si los actos dolosos no implican precisamente eso. O si la culpa inexcusable no significa la inejecución negligente y temeraria.

Al respecto, al definir la forma como se sancionará al OTM, el artículo 17 precisa que éste responderá por pérdidas, daños o averías durante la ejecución del contrato, "a menos que pruebe que él, sus dependientes $o$ agentes o cualquier otra persona que se hallare a su servicio, adoptaron las medidas que razonablemente podían exigirse para evitar el hecho y sus consecuencias".

Por la forma en que está redactado ese artículo, parece que en principio adopta la tesis de la responsabilidad por riesgo profesional del OTM, pues lo hace responsable sin ambages de los daños que ocurran mientras 
los efectos están bajo su custodia; pero más adelante varía todo el concepto, al permitir la excepción basada en el comportamiento razonable o diligente, que importa definitivamente la connotación de culpa.

Sin embargo, bajo esta forma de definición, no se separan los casos de culpa leve, grave o dolosa, colocando al porteador en la misma posición, tanto si su comportamiento es de uno u otro tipo, pues en cualquier caso solamente responderá por el máximo que la ley le ha señalado.

Hubiese sido recomendable que se adoptase, conjuntamente con una limitación de este tipo, la definición exonerativa que se dio al transportista terrestre ya vista, en el sentido de que sólo queda exonerado por la concurrencia de la fuerza mayor, caso fortuito, acto del cargador o vicio propio de la cosa, así como establecer que por los hechos dolosos o culposos graves se pierda el paraguas de la protección limitativa, de la misma manera como lo señala la Convención de Varsovia para los casos de responsabilidad aeronáutica.

Ahora bien, ¿qué significa esta responsabilidad cuantitativamente hablando? Conforme ya hemos señalado anteriormente en el desarrollo de este mismo artículo, los DEG -o SRD, como los llama equivocadamente el decreto legislativo 704- tienen aún ahora, en la actualidad, a los veinte años de su aparición, el mismo valor inicial, esto es, aproximadamente la equivalencia de US\$1,2, lo cual significa que las 666,67 unidades de cuenta alcanzan escasamente a US\$ 800 .

En palabras simples, resulta que un operador de transporte multimodal que es responsable por las consecuencias de dos formas diferentes de acarreo de los bienes que recibe, responde, como máximo, ante un usuario, por una suma que es diez (10) veces menor que la responsabilidad que frente a éste tiene a su vez un transportista por agua. Por supuesto, la situación será más grave si se trata de un transportista terrestre, que está obligado a responder por el valor comercial del bien, incluyendo seguros y flete, o del aéreo, que dependerá del peso que tenía el artículo. Bastaría, para caer en el ridículo, señalar que un bulto que pesara solamente 4,500 kilogramos ya significará para el transportista aéreo una responsabilidad mayor que la máxima que tendría el operador de transporte multimodal.

La disposición que existe sobre el particular precisa, en su artículo 18, que es el OTM el único que responde frente al usuario como si los actos de los terceros que utilizó o contrató hubiesen sido actos propios, pero no le limita de la misma manera la forma en que realizará el reclamo contra este porteador del segmento, propiciando de esa manera la posibilidad de un enriquecimiento de parte del OTM, a riesgo del empobrecimiento del usuario o de su asegurador. 
Dentro de este esquema limitativo es incomprensible la excepción que incluye el artículo 21 de la ley que se comenta, por la cual se establece que si en el contrato de porteo intermodal no se incluye «el porte de mercancías por mar o por vías de navegación interior", la responsabilidad del OTM ya no será el máximo de 666,67 unidades de cuenta, sino que se establecerá en función del peso bruto de las mercaderías, fijándose para el efecto 8,33 SRD o DEG por kilogramo grueso, o, lo que es lo mismo, US\$ 9,99.

La exoneración del quantum resarcitorio que el OTM debe pagar por daños o pérdidas de bienes recibidos, no solamente funcionará, según el artículo 17, por acreditarse la utilización de medidas razonables, sino que además el artículo 26 incluye cinco nuevas causales exonerativas (fuerza mayor o caso fortuito, vicio propio, deficiencia de embalaje, cumplimiento de normas legales, $y$ hecho del expedidor o consignatario). En principio creemos que pudieron haberse resumido en tres causales: caso fortuito o fuerza mayor, vicio propio y hecho del expedidor o consignatario. Pero además esta norma señala, que aun en presencia de estas causales de exoneración el OTM será responsable por el «eventual agravamiento de las pérdidas o daños, cuando se compruebe que exista negligencia respecto de dichas circunstancias».

En otras palabras, para el legislador es posible establecer una diferencia en la responsabilidad entre el hecho generador del daño y las consecuencias agravantes de éste; sin embargo, la pregunta que flota es de qué manera podemos hacer un punto de inflexión para separar el momento económico en que los daños dejaron de no ser responsabilidad del OTM y pasaron a ser de costo.

Estimamos que esta declaración es un tratamiento más lírico que prácrico, y conlleva definitivamente una imposibilidad de determinación, por lo que si alguna vez un OTM puede ser condenado por esta causa, condena a la cual siempre podrá oponer su limitación de responsabilidad de US $\$ 800$ como máximo, no será posible nunca fijar el momento exacto y la cuantificación de esas acciones condenatorias.

La legislación, a través del artículo 17, también imputa al OTM responsabilidad por el retraso, o lo que es conocido legislativamente como el cumplimiento tardío de su obligación. Sin embargo, ese efecto inicial es mitigado totalmente y casi ridiculizado al señalarse en el artículo 22 que existe una segunda limitación de responsabilidad para este hecho, diferente de la anterior. En ese caso de daños por retraso la ley los limita al valor del flete acordado por el transporte multimodal, sin justificarse realmente la razón de estimar únicamente ese monto contractual como el que deba ser objeto de satisfacción por el porteador. 


\section{Conclusiones}

La inferencia que se desprende del análisis rápido de las normas aplicables para el transporte internacional de mercancías en el Perú, es que luego de noventa y dos años de vigencia de la legislación comercial los porteadores se han visto cada vez más beneficiados con la limitación de responsabilidad. La disminución cuantitativa es notoria, y no se ha tomado siquiera en cuenta que una figura como la de la limitación de este tipo solamente puede ir acompañada de una imputación de responsabilidad netamente objetiva, que además siempre fue desde el Derecho romano la forma típica de responsabilizar al porteador por los daños que causaba a terceros.

Es importante revisar la actual legislación de la materia, uniformarla y estudiar la conveniencia de establecer criterios rectores paritarios que definitivamente beneficien a los usuarios y no solamente a unos cuantos, que curiosamente al final no resultan siendo peruanos sino transportistas extranjeros, quienes en sus países además gozan de beneficios de diversa índole, situación que no es compatible con las normas de liberalidad y competitividad comercial que se preconiza. 